\title{
Politique
}

Politique

\section{À la rescousse des banques de Marc Chabot (préface d'André Blais), Sillery, Presses de l’Université du Québec, 1991, 247 p.}

\section{Pierre P. Tremblay}

Numéro 23, hiver 1993

Tendances de la science politique au Québec

URI : https://id.erudit.org/iderudit/040759ar

DOI : https://doi.org/10.7202/040759ar

Aller au sommaire du numéro

Éditeur(s)

Société québécoise de science politique

ISSN

0711-608X (imprimé)

1918-6584 (numérique)

Découvrir la revue

Citer ce compte rendu

Tremblay, P. P. (1993). Compte rendu de [À la rescousse des banques de Marc Chabot (préface d'André Blais), Sillery, Presses de l'Université du Québec, 1991, 247 p.] Politique, (23), 234-238. https://doi.org/10.7202/040759ar d'utilisation que vous pouvez consulter en ligne.

https://apropos.erudit.org/fr/usagers/politique-dutilisation/ 


\section{A la rescousse des banques}

de Marc Chabot (Préface d'André Blais), Sillery, Presses de I'université du Québec, 1991, 247 pages.

Le titre est résolument accrocheur. Les difficultés des empires financiers font recette de nos jours et elles renforcent chez le commun des mortels l'idée d'une justice immanente, même pour entreprendre la lecture de cette thèse de doctorat que son auteur a légèrement remaniée afin de la rendre accessible à un public élargi.

D'instinct, je partage avec Marc Chabot l'opinion que le sauvetage des entreprises par les gouvernements ou par certaines de leurs créatures est une question importante. Ses dimensions sociale et politique doivent, à mon avis, être débattues au même titre que ses enjeux économiques et financiers. Quels motifs poussent l'État à intervenir en faveur 
de certains et pourquoi, dans d'autres cas, choisit-il de ne pas bouger? Voilà des questions qui tarabustent autant l'homme de la rue que le spécialiste des affaires publiques. Le livre de Chabot a voulu jeter quelques éclairages nouveaux sur ce problème de grande actualité. II a scruté, pour ce faire, les dossiers d'entreprises dont la faillite appréhendée a mobilisé le secours de l'État ainsi que les dossiers d'entreprises dont les appels à l'aide furent ignorés.

Le livre se divise en cinq chapitres. Dans le premier, l'auteur présente son objet d'étude : la faillite et l'insolvabilité des entreprises. Nous sommes, d'entrée de jeu, conviés à un tour d'horizon de la recherche en la matière. On y apprend dès lors qu'en dépit de la croissance rapide du phénomène, les études canadiennes en droit de la faillite sont peu nombreuses. Ce qui ne semble pas être le cas en sciences administratives, particulièrement en comptabilité et en finance. Marc Chabot souligne d'ailleurs que l'intérêt observé en science de la gestion nous a valu quelques modèles de prédiction fort valables dont le plus connu serait celui de $E$. Altman publié en 1968. La science économique a aussi la faillite et l'insolvabilité comme objets de recherche. La perspective, aux dires de l'auteur, y serait même plus large qu'en science administrative. Cependant, c'est vers la science politique qu'il se tourne pour entreprendre sa recherche. II justifie son choix par le rôle central accordée à l'État dans son analyse et par le fait qu'il retient l'affrontement des intérêts comme fondement de son hypothese.

Au chapitre 2, Chabot se livre à une revue de la littérature. De facture très classique, cette synthèse met résolument l'accent sur les travaux économiques de Trebilcock, sur certaines études de cas (Ville de New York, Chrysler, Dome Petroleum, etc.l, et sur les études générales, notamment celle d'André Blais traitant de la rationalité politique de l'aide à l'entreprise et celle de Zysman sur les systèmes financiers. L'exercice est un peu court et ne permet pas de dégager les grandes idées en la matière. Le cadre théorique de la recherche exposé au chapitre suivant va d'ailleurs souffrir de la faiblesse de la revue de la littérature. En effet, le libellé de l'hypothèse de travail constitue 
davantage un constat qu'une explication raisonnée du traitement de faveur accordé aux institutions financières dans le cas de certaines faillites d'entreprises. L'anémie du cadre théorique aura par la suite des effets destructeurs sur la qualité et la pertinence de l'étude. Il est frappant de constater que la formulation de l'hypothèse ( «le sauvetage d'entreprises insolvables par le gouvernement bénéficie plus aux banques et aux institutions financières qu'aux travailleurs, aux fournisseurs ou encore aux actionnaires") évacue toute dimension politique que les travaux de Blais auraient dû lui apprendre. Pour une recherche de science politique, il y a là un oubli plutôt déplorable. Marc Chabot consacre son quatrième chapitre aux études de cas proprement dites. Parmi les dossiers analysés, certains furent célèbres en leur temps, c'est-à-dire qu'ils ont eu une bonne couverture de presse. On parle alors de Dome Petroleum ou de MasseyFerguson par exemple. D'autres furent moins célèbres; ce fut le cas de CCM et d'Electrohome. Pour fins de comparaison et de rigueur scientifique, Marc Chabot traite, dans son ouvrage, de cas où il y eut sauvetage et de cas où aucun sourien n'est venu du côté du gouvernement fédéral. Enfin, dans un cinquième chapitre et dans la conclusion, l'auteur expose les résultats de sa recherche et en tire certains enseignements.

Cet ouvrage s'affiche comme une analyse de politique. C'est d'ailleurs ce que confirme sans ambiguïté la préface rédigée par André Blais. Je ne suis pas d'accord avec cette prétention. II me semble qu'une collection d'études de cas ne saurait constituer une véritable analyse de politique en l'absence de données fondamentales. À titre d'exemple, on ne sait toujours pas, au sortir du bouquin, quelle est la politique du gouvernement fédéral en ce qui regarde les entreprises en difficultés. II doit bien exister quelque part un bout de papier qui en fait état. Peut-être même qu'un ou deux hauts fonctionnaires impliqués auraient pu tracer les grandes lignes de la pensée gouvernementale sur ce sujet ! Qu'en est-il aussi des objectifs poursuivis, des programmes ainsi que des résultats factuels ou désirés? On dit bien, dans ce bouquin, que l'aide aux entreprises insolvables est un élément de la politique industrielle. Fort bien. Malheureusement, l'auteur se limite à ce cliché et choisit de ne pas 
s'aventurer plus avant dans un exposé qui aurait do être le point d'ancrage de son cadre théorique. Donc, sans toile de fond, on a droit à une hypothèse tronquée qui ne dit rien de la problématique du sauvetage des entreprises et qui ne trace pas de piste à explorer. Une hypothèse complète aurait situé ce constat par rapport à des éléments identifiables de la politique industrielle (rôle moteur des banques en matière d'investissements...) et aurait fourni au lecteur une interprétation du résultat. Cette carence majeure empêche, à mon avis, l'auteur d'atteindre son objectif principal qu'il décrit (page 52) comme étant d'appréhender l'influence politique des banques canadiennes. Encore que $c^{\prime}$ est une chose de cerner le poids politique des institutions financières; $c^{\prime}$ en est une autre d'en révéler les enjeux. Bref, la méthodologie de l'analyse de politique, tout comme celles utilisées pour faire des évaluations de politiques ou des évaluations de programmes, requiert de la part de l'analyste une recherche documentaire de base afin de préciser le degré de faisabilité de son étude. Chabot a, selon toutes les apparences, omis de faire cette démarche préalable et indispensable. S'il l'a fait, il n'en souffle pas mot. Au départ, son ouvrage est, pour cette raison, un produit bancal.

À mon avis, cet ouvrage manque de profondeur. II se borne à confirmer quelques soupçons populaires sur l'importance hiérarchique des entreprises et sur le rôle clé des banques en matière d'investissement. On se serait attendu à un peu plus d'originalité et à un effort théorique plus ferme. Ce n'est manifestement pas le cas. L'œuvre renferme aussi de ces petits irritants qui, à la longue, tombe carrément sur les nerfs rendant la lecture insupportable. Le style de l'auteur transpire l'exercice académique; l'écriture est lourde, redondante et saccadée. En général, je suis bon lecteur comme on dit "bon public" mais j'avoue avoir fait des efforts soutenus pour trouver de l'intérêt au livre de Chabot. Somme toute, je déplore qu'un sujet aussi aguichant et aussi porteur de recherche ait été trop brièvement traité et si mal écrit. De plus, une analyse de politique demande un travail plus complexe et plus fouillé qu'une simple lecture de dossier. Marc Chabot s'est, malheureusement, laissé enfermer dans une 
approche encore trop comptable. Pour tout dire, je préfère $L a$ part du lion de Linda McQuaig.

Pierre Tremblay

Université du Québec à Montréal 